\title{
Early detection of osteoarthritis in the rat with an antibody specific to type II collagen modified by reactive oxygen species
}

Anne Gigout ${ }^{1}$, Donata Harazin ${ }^{1}$, Louise M. Topping ${ }^{2}$, Didier Merciris ${ }^{3}$, Sven Lindemann ${ }^{1}$, Christian Brenneis ${ }^{1}$ and Ahuva Nissim ${ }^{2 *}$ id

\begin{abstract}
Background: Osteoarthritis $(\mathrm{OA})$ is a disease of the whole joint, with articular cartilage breakdown as a major characteristic. Inflammatory mediators, proteases, and oxidants produced by chondrocytes are known to be responsible for driving cartilage degradation. Nevertheless, the early pathogenic events are still unclear. To investigate this, we employed an antibody that is specific to oxidative post-translationally modified collagen type II (anti-oxPTM-CII) to detect early cartilage pathogenic changes in two rat models of OA.

Methods: The animals underwent surgery for destabilization of the medial meniscus (DMM) and were sacrificed after 3,5, 7, 14, and 28 days. Alternatively, anterior cruciate ligament transection with partial meniscectomy (ACLT+pMx) was performed and animals were sacrificed after 1, 3, 5, 7, and 14 days. Joints were stained with toluidine blue and saffron du Gatinais for histological scoring, anti-oxPTM-Cll, and anti-collagen type X antibodies (anti-CX).

Results: We observed positive oxPTM-Cll staining as early as 1 or 3 days after ACLT+pMx or DMM surgeries, respectively, before overt cartilage lesions were visible. oxPTM-CII was located mostly in the deep zone of the medial tibial cartilage, in the pericellular and territorial matrix of hypertrophic chondrocytes, and co-localized with CX staining. Staining was weak or absent for the lateral compartment or the contralateral knees except at later time points.

Conclusion: The results demonstrate that oxidant production and chondrocyte hypertrophy occur very early in the onset of OA, possibly initiating the pathogenic events of OA. We propose to use anti-oxPTM-CII as an early biomarker for $\mathrm{OA}$ ahead of radiographic changes.
\end{abstract}

Keywords: Reactive oxygen species, Osteoarthritis, Hypertrophy, Collagen type II, Collagen type X

\footnotetext{
*Correspondence: a.nissim@qmul.ac.uk

${ }^{2}$ Barts and the London School of Medicine and Dentistry, Queen Mary University of London, Chaterhouse Square, London EC1M 6BQ, UK

Full list of author information is available at the end of the article
}

(c) The Author(s). 2021 Open Access This article is licensed under a Creative Commons Attribution 4.0 International License, which permits use, sharing, adaptation, distribution and reproduction in any medium or format, as long as you give appropriate credit to the original author(s) and the source, provide a link to the Creative Commons licence, and indicate if changes were made. The images or other third party material in this article are included in the article's Creative Commons licence, unless indicated otherwise in a credit line to the material. If material is not included in the article's Creative Commons licence and your intended use is not permitted by statutory regulation or exceeds the permitted use, you will need to obtain permission directly from the copyright holder. To view a copy of this licence, visit http://creativecommons.org/licenses/by/4.0/ The Creative Commons Public Domain Dedication waiver (http://creativecommons.org/publicdomain/zero/1.0/) applies to the data made available in this article, unless otherwise stated in a credit line to the data. 


\section{Background}

Osteoarthritis (OA) is one of the leading causes of reduced quality of life worldwide, due to the associated chronic pain and various degrees of disability. Although OA affects all the tissues of the articular joint, degradation and loss of articular cartilage is a central feature $[1,2]$. Cartilage degradation in OA results from a disruption in homeostasis due to activation of the chondrocytes by various factors that promote the production of matrix degrading enzymes, in excess of the capacity of the chondrocyte to replace damaged and degraded matrix components. The factors that activate chondrocytes to promote matrix degradation include excessive and abnormal mechanical loading, pro-inflammatory cytokines, and chemokines, as well as Wnt ligands and factors activating the innate immune system $[1,3]$. Many of these OA factors stimulate chondrocytes to produce reactive oxidants (ROS). ROS are utilized as secondary messengers in mediating intracellular signaling events that regulate expression of matrix degrading enzymes [4, 5] and pro-death signaling pathways, thus compromise chondrocyte integrity and promote cartilage damage [6]. The most abundant ROS produced by chondrocytes include superoxide, hydrogen peroxide, the reactive nitrogen species nitric oxide, and the nitric oxide derived product peroxynitrite [2].

Experimental OA models induced by joint instability have been highly valuable in identifying key pathogenic pathways in disease and for validating new treatments. They produce robust degradation of the articular cartilage and changes in the subchondral bone and can be used to investigate pain-like symptoms. Widely used models of animal OA involve surgically induced instability of the knee [7]. These models are characterized by an acute injury to the joint that causes mechanical instability, resulting in OA. Many types of operations on various animals have been developed, including cruciate or collateral ligament transections and partial or total meniscectomies on dogs, goats, rabbits, and rodents. Most of the studies detect mild changes in the articular cartilage at 2 to 4 weeks post-operatively. For example, in the anterior cruciate ligament transection (ACLT) model, cartilage destruction is seen 2-4 weeks after surgery [8]. Alternatively, when OA is induced by destabilization of the medial meniscus (DMM), structural-change progression is slower [9-11]. Currently, the size of the animal precludes prospective assessment of disease by conventional radiographic approaches, and disease is assessed by serial histology of the joint, which is time consuming, costly, and requires large number of animals as they need to be culled at each experimental time point under investigation. Powerful non-invasive small-animal imaging techniques for longitudinal studies are therefore highly desirable for preclinical validation studies as well as for detection and monitoring of early OA in patients.

We previously developed a panel of human single chain fragment variable (scFv) that binds specifically to oxidative post-translationally modified collagen type II (oxPTMCII) [12]. We showed that anti-oxPTM-CII (i) binds specifically to arthritic cartilage from patients with RA and $\mathrm{OA}$; (ii) stains cartilage in murine models of inflammatory arthritis (antigen induced arthritis (AIA) and OA, namely DMM); (iii) localizes in the arthritic joint in vivo in a mouse model of AIA and DMM following systemic administration of labeled anti-oxPTM-CII with Alexa Fluor 680 or Cy5.5 [12, 13]; and (iv) was able to target therapeutic scaffolds specifically to arthritic joint [14].

In the current study, we evaluate longitudinally the presence of oxPTM-CII staining in early OA in two rat models: DMM and ACTL+pMx. Our goal was to possibly unveil some of the very early events in OA and evaluate the possibility to detect the initiation of the disease before the appearance of cartilage lesions. Rats were sacrificed 3, 5, 7, 14, and 28 days after DMM and 1, 3, 5, 7, and 14 days after ACTL+pMx surgery and the lateral and contralateral knees were stained with toluidine blue and saffron du Gatinais, or for oxPTM-CII and type X collagen (CX). OxPTM-CII and CX were detectable already at the earliest time points in the medial tibial cartilage and strongly co-localized together. We conclude that (i) ROS production and increased type X collagen expression are early events in $\mathrm{OA}$ and prefigure cartilage lesions and (ii) anti-oxPTM-CII detection could be a powerful tool to detect initiation of OA.

\section{Method}

\section{Antibody preparation}

Anti-oxPTM-CII scFv was expressed in HB2151 bacteria as described [15]. ScFv was converted to full length antibody by cloning $\mathrm{V}_{\mathrm{H}}$ domain into pFUSEss-CHIg-hG1e3 and $\mathrm{V}_{\mathrm{L}}$ domain into pFUSEss-CLIg-hk (InvivoGen). Plasmid DNA was isolated using a QIAFilter Plasmid Maxi Kit according to the manufacturer's instruction (QIAGEN). Following transient expression in Expi293F Expression System according to the manufacturer's instructions (Thermo Fisher Scientific), supernatants were collected and purified using protein A Sepharose CL-4B (GE Healthcare). The ability to retain specific binding of anti-oxPTM-CII over native CII was assessed by ELISA as described [12].

\section{Animal models}

Male Lister Hooded (Crl:LIS) outbred SPF rats (aged 89 weeks and within 150-175 g weight range, Charles River) were housed in colony cages as described [16] with 48 rats/cage at the start of the study. After 4 weeks of acclimatization, rats underwent surgery under 
anesthesia. Anterior cruciate ligament transection with partial meniscectomy (ACLT+pMx) was performed as described elsewhere [16] with the exception that only $50 \%$ of the meniscus was removed. For destabilization of the medial meniscus (DMM), a skin incision was made from distal the patella proximal to the tibial plateau (of the right joint). The muscle layer was opened in knee flexion with a scalpel and prepared to visualize the medial meniscus tendon which was ligated using scissors. Finally, the joint capsule, associated muscles and connective tissue were sutured in layers. For postsurgical analgesia, rats received meloxicam $(0.5 \mathrm{mg} / \mathrm{kg}$ s.c.; Metacam injection solution, Boehringer Ingelheim). At the specified time points following surgery, rats $(N=9-10$ per time point) were humanely euthanized by transthoracic heart puncture under isoflurane anaesthetization.

\section{Histology and scoring}

The ipsilateral knees of all animals and contralateral knees of two animals per time points $(N=10)$ were fixed for 7 days in paraformaldehyde (VWR) $4 \%$ in phosphatebuffered saline (PBS, VWR) and decalcified for 6 weeks in formic acid (Sigma-Aldrich) $4 \%$ in PBS and embedded in paraffin. Coronal sections of $7 \mu \mathrm{m}$ (including medial tibial plateau, femur condyle, and menisci) were cut with a microtome within the weight-bearing area. Every 35th section was collected. The sections were deparaffinized and rehydrated, stained with toluidine blue (VWR, $0.05 \%$ in PBS, $6 \mathrm{~min}$ ) and saffron du Gatinais (Morphisto $\mathrm{GmbH}$, diluted 1:3 in absolute Ethanol, $1 \mathrm{~min}$ ). Slides were then dehydrated, coverslipped, and after drying digitized using slide scanner SCN400 (Leica Microsystems). For histopathological grading, we adapted the modified Mankin score described in [17] and recommended by the OsteoArthritis Research Society (OARSI) for guinea pig. The changes we made to this score had the purpose to make it usable for several species, to be able to compare results obtained with various types of animals. In the present work, we used it to describe rat OA. Our score has a maximum SUM score of 28. Sub-scores are described in supplementary material Table S1. Scanned sections were analyzed by two independent observers and the most severe lesion within the weight-bearing area for each rat was selected for evaluation. For each subscore, the values from two consecutive sections were averaged to determine overall values for each animal.

\section{Immunostaining}

Single or double staining for type X collagen (CX) and ROS-modified type II collagen (oxPTM-CII) were applied. For the ACLT+pMx study, two single staining were conducted, one for CX and one for oxPTM-CII. For the DMM study, to evaluate the co-localization of
$\mathrm{CX}$ and oxPTM-CII a double staining was first performed. However, because the blue staining of CX was very dark and both staining overlapped, it was difficult to visualize clearly the brown staining of the oxPTMCII. For this reason, for the DMM study, an additional single staining for oxPTM-CII was conducted.

For the CX staining, the sections were first deparaffinized and rehydrated. An epitope retrieval using Proteinase K (Leica Biosystems) [18] was performed. Sections were subsequently incubated with a monoclonal mouse antibody specific for Collagen X (\#1-CO097-05, Quartett) diluted 1:50, for $30 \mathrm{~min}$ at room temperature. For the detection, the Leica Polymer Refine Red Detection System (\#DS9390, Leica Biosystems) was used where the RED dye was substituted with NBT/BCIP (\#ab7468, Abcam). The type $\mathrm{X}$ collagen staining was developed using a fully automated immunohistochemistry stainer (Bond III, Leica Microsystems).

For the staining of oxPTM-CII, epitope retrieval was performed with pepsin. Sections were first equilibrated in $\mathrm{HCl}$ (Merck $\mathrm{KGaA}$ ) $0.02 \%$ in $\mathrm{ddH}_{2} \mathrm{O}, 37^{\circ} \mathrm{C}, 15 \mathrm{~min}$ and digested with $15 \mathrm{mg} / \mathrm{mL}$ pepsin (Merck $\mathrm{KGaA}$ ) in $\mathrm{HCl} 0.02 \%, 37^{\circ} \mathrm{C}, 45 \mathrm{~min}$ [19]. The sections were incubated with the anti-oxPTM-CII antibody $6.5 \mu \mathrm{g} / \mathrm{mL}$ overnight at $4{ }^{\circ} \mathrm{C}$ and detected using Polink-2 Plus HRP human IgG with 3,3'-diaminobenzidin (DAB) (\#D88, GBI Labs).

For both staining protocols, negative controls were performed where the primary antibody was omitted.

$\mathrm{CX}$ and oxPTM-CII staining were quantified in a region of interest (ROI) selected on the tibial medial plateau using the image analysis software, Calopix ${ }^{\circ} \mathrm{v}$ 4.1.0.3 (Tribvn, France). Positive areas for each staining were expressed as the percentage of the total cartilage area in the ROI. The ROI was placed where lesions develop and was $1 \mathrm{~mm}$ long (the width corresponded to cartilage thickness). Because the growth plate was found to be positive for both oxPTM-CII and CX, sections that were not or only weakly stained in this area were excluded from the analysis.

\section{Statistical analysis}

Data were analyzed for their normality with the D'Agostino Person or the Shapiro-Wilk normality test (for $n<$ 8). For most of the data, several groups did not follow a normal distribution and the Kruskal-Wallis test corrected for multiple comparison with a Dunn's test was applied. For the oxPTM-CII quantification results in the DMM and ACLT+pMx model and the CX quantification in the ACLT model, all groups followed a normal distribution and in this case, a one-way ANOVA corrected for multiple comparison with a Dunnet test was applied. GraphPad Prism v8.4.2 was used. 


\section{Results}

DMM surgery affects more profoundly and ACLT+pMx more strongly the medial tibial compartment

After both DMM and the ACLT+pMx surgeries, osteoarthritis develops mostly in the medial tibial plateau and this area was scored according to the histochemicalhistological scoring system modified from [17]. For the DMM model, animals were sacrificed at days 3, 5, 7, 14, and 28. Because ACLT with meniscectomy results in more severe OA compared to DMM [9], earlier time points were chosen for this model and animals were sacrificed at days $1,3,5,7$, and 14. After DMM surgery, loss of matrix staining in cartilage was apparent already after 3 days as illustrated on Fig. 1a and on the matrix staining sub-score (Figure S1) but cartilage defects appeared only at day 28 in most of the animals (Fig. 1e and sub-score cartilage structure in Figure S1). Most of the other sub-scores such as cellularity, alteration of the tidemark and thickening of the subchondral bone started to be evident at day 14 . Small to medium-sized osteophytes were observed in a minority of animals 14 or 28 days after the surgery. As a result, the total histological score was significantly elevated at day 14 and 28 in comparison to the contralateral medial tibial plateau (Fig. 1)
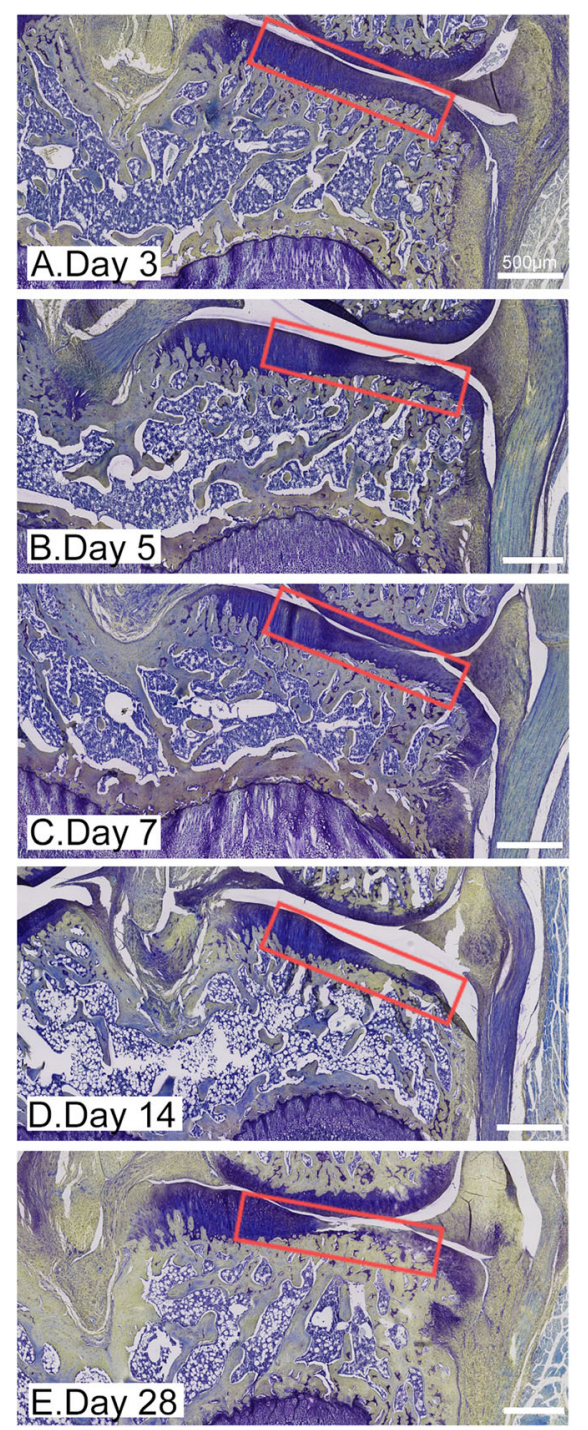

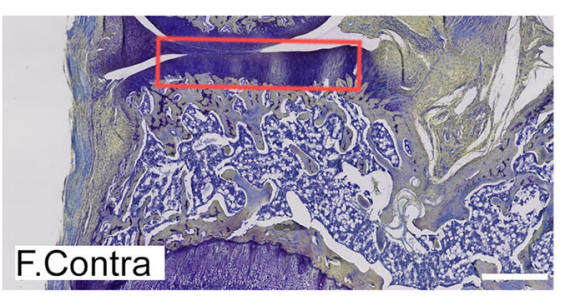

Total histological score: DMM

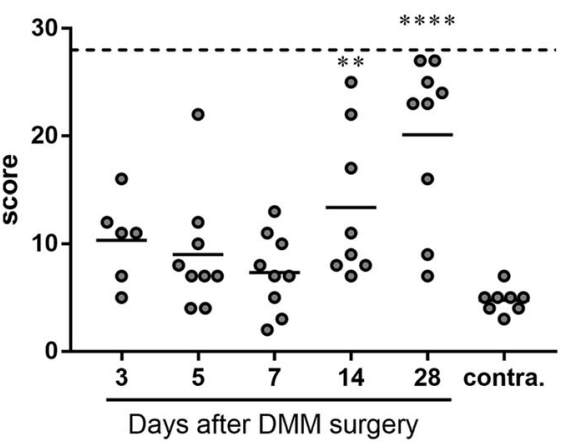

Total histological score: ACLT+pMx

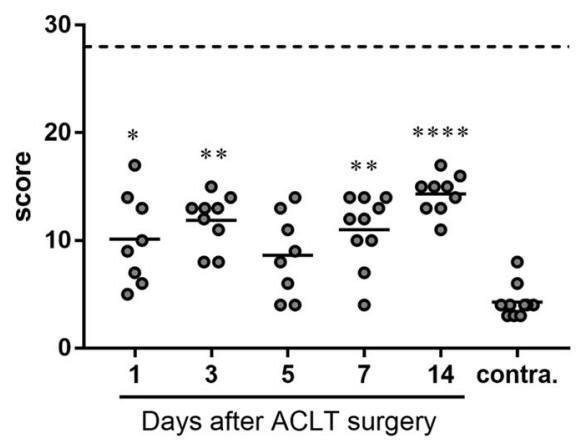

Fig. 1 Histological scoring of OA for the medial tibial plateau in the ACLT+pMx and DMM models. Rats underwent ACLT+pMx or DMM surgery and were sacrificed at various time points ( $N=9-10$ rats per time points). The ipsilateral or contralateral knees were taken for histological analysis Slides were stained with toluidine blue and saffron du Gatinais and scored as detailed in the "Method". Histological sections for the DMM model are shown with the region selected for scoring marked in red. The total histological score is shown for both models. Data on the graphs represent the total score for each animal $(N=9-10)$ and the mean for each time point and for the selected contralateral knees. Double asterisks and quadruple asterisks mean significantly different from contralateral with $p<0.01$ or $p<0.0001$, respectively 
for this model. After the ACLT+pMx surgery, the total histological score (Fig. 1) was significantly elevated from the first day compared to the contralateral knee and this was mainly driven by the cartilage structure and the matrix staining sub-scores (Figure S2). The other subscores were barely affected, and no osteophyte was observed. The comparison of both models at days 3-14 shows that the total histological scores were higher (except at day 5) for the ACLT+pMx model, and this seems to be mostly driven by the higher sub-scores for cartilage structure and matrix staining. However, more sub-scores were affected in the DMM compared to ACLT+pMx.

\section{OxPTM-CII and CX signal in the deep zone are early markers of $O A$}

ACLT+pMx and DMM knees were stained with antioxPTM-CII and antibody specific to CX (staining for the medial tibial plateau is shown in Fig. 2 and for the lateral tibial plateau in Figure S3). On the medial compartment (Fig. 2), for both the ACLT+pMx and DMM knees, a strong CX staining is observable in the deep zone located where matrix loss or cartilage damages were also visible. Otherwise, in the rest of the deep zone, the staining was weak all along the tidemark. Similarly, most of the cartilage was negative for oxPTM-CII but a positive staining was evident in the deep zone in the region where cartilage degradation occurred. Interestingly, both staining were already observable at early time points, before visible cartilage damage started to develop. Both staining were also particularly strong where hypertrophic chondrocytes in the deep zone were visible. In addition, in the DMM model at day 28 , more severe damage could be observed. In this case, oxPTM-CII and CX staining extended beyond the deep zone at the damage site and the full depth of cartilage was positive for CX.

We also looked at the lateral tibial compartment of the operated knees (Figure S3) and the contralateral knees (Figure S4). Both the ACLT+pMx and DMM models showed weak or no visible staining in the lateral tibial compartment at early time points (days 1 or 7 or days 3 or 14, respectively) for CX and oxPTM-CII irrespective of the histological score obtained on the medial side (indicated in brackets Figure S3A). However, at day 14 in the ACLT+pMx model and day 28 in the DMM model signal for both collagens appeared. Contrary to the medial tibial cartilage, the staining was not restricted
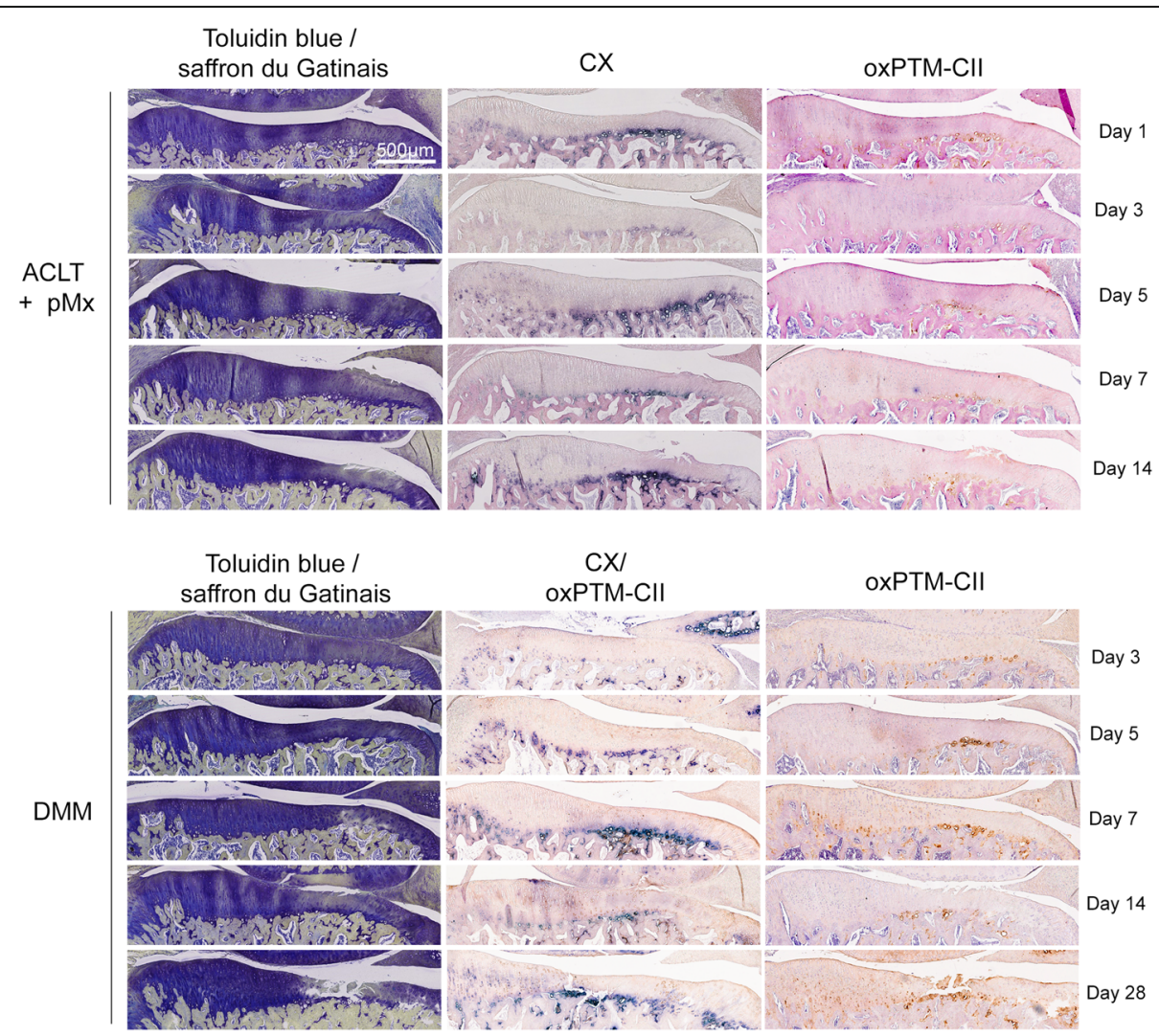

Fig. 2 CX and oxPTM-Cll staining of the medial tibial plateau cartilage in the ACLT+pMx and DMM models. Representative results for toluidine blue and saffron Gatinais as well as type X collagen (blue, CX) and oxPTM type II collagen (brown, oxPTM-CII) immunostainings are shown. For the ACLT+pMx, study single immunostainings were realized. For the DMM study, a CX and oxPTM-Cll double staining was performed as well as an oxPTM-Cll collagen single staining 
to a specific location in the cartilage. These results illustrate that at later time points the disease progressed to the lateral side of the operated joint. The lateral tibial compartment was scored as well (Figure S3B) and the histological scores were low in all groups and no difference could be observed to the contralateral lateral tibial compartment at all time point tested.

Similarly, the contralateral knees (Figure S4) showed only a weak CX and oxPTM-CII staining at day 1 or 3 in the ACLT+pMx or DMM models respectively but an increased staining intensity was visible on the medial side for both CX and oxPTM-CII after ACLT+pMx at day 14 and for oxPTM-CII after DMM at day 28. As for the ipsilateral knee, CX and oxPTM-CII were strictly localized in the deep zone around the large hypertrophic chondrocytes in the medial tibial compartment, and the staining appeared more diffuse in the lateral tibial compartment.
Finally, condylar cartilage was found to be mostly negative for oxPTM-CII (not shown) but started to be positive when large damages develop on the tibial side (see example on Fig. 3d, e). Condylar cartilage was also weakly positive for $\mathrm{CX}$, which was strictly localized in the deep zone. A stronger CX staining appeared with the progression of the disease but at later time points compared to the tibial cartilage (data not shown).

\section{OxPTM-CII and CX staining partially co-localize}

Higher magnifications of the medial tibial cartilage from DMM knees are shown in Fig. 3 for cartilage with matrix staining loss but no defect (Fig. 3a, b) or for cartilage with a small (Fig. 3c) or a large defect (Fig. 3d). An example for condylar cartilage is also shown (Fig. 3e). Only the DMM knees are shown as the double staining for $\mathrm{CX}$ and oxPTM-CII enabled to better determine whether both markers co-localized. In the cartilage with

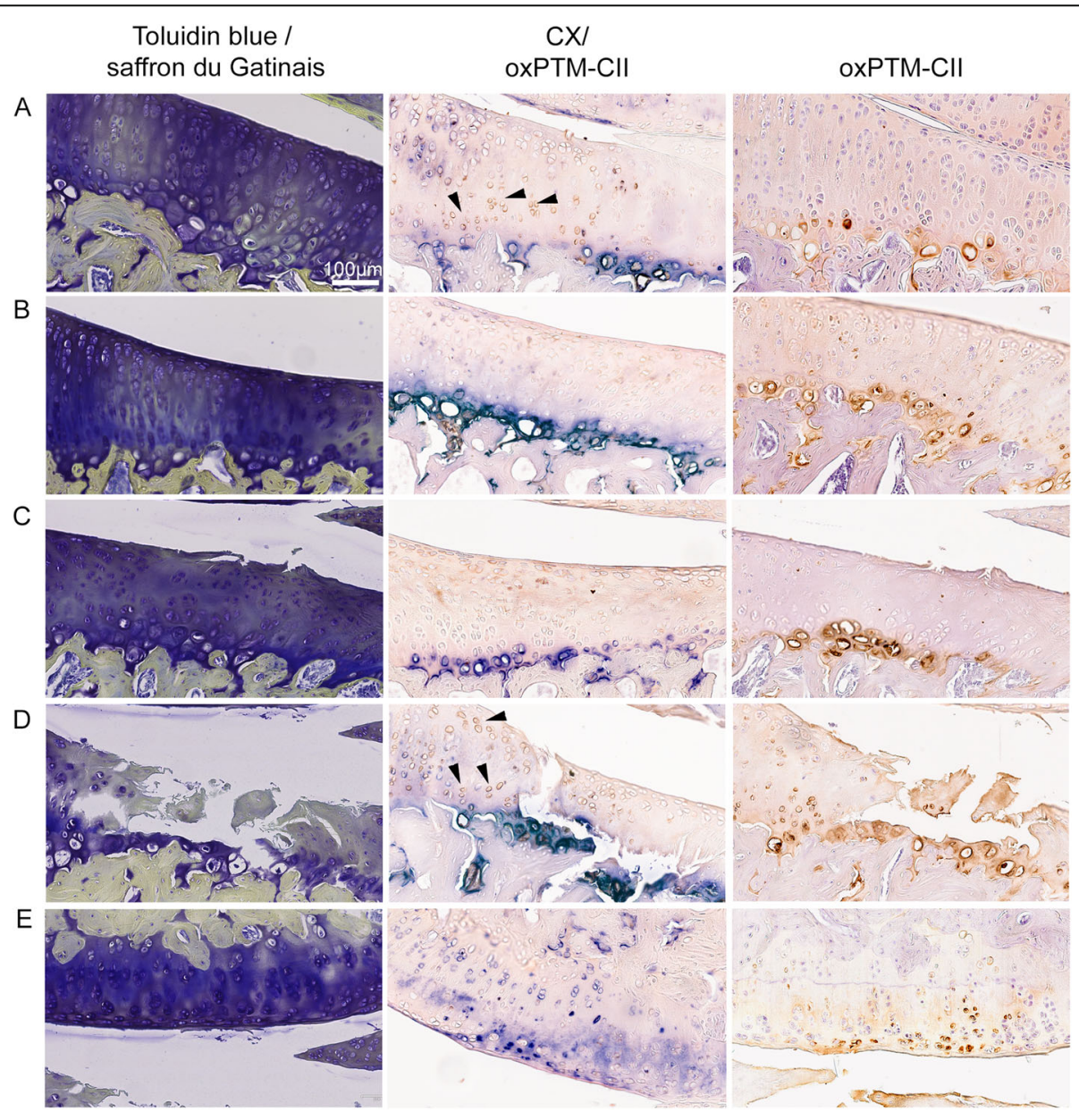

Fig. 3 Partial co-localization of CX and oxPTM-Cll staining in the cartilage of the medial tibial plateau and condyle in the DMM model. Toluidin blue and saffron du Gatinais, type X collagen (blue, CX) and oxPTM type II collagen (brown, oxPTM-CII) staining are shown for cartilage of the medial tibial plateau presenting different levels of degeneration: $\mathbf{a}, \mathbf{b}$ loss of matrix staining but no defect (both from day 14) and $\mathbf{c}$, $\mathbf{d}$ cartilage with a small and a large defect, respectively (from day 5 and 28, respectively). One example of staining for cartilage form the medial condyle is shown in e. Black arrows show examples of chondrocytes positive for OxPTM-CII but negative for CX 
no apparent defect, $\mathrm{CX}$ and oxPTM-CII staining colocalized in the deep zone mostly in the pericellular and territorial matrix of the hypertrophic chondrocytes. In panel A, chondrocytes that were positive for oxPTMCII but negative for CX can also be observed (see arrows). In cartilage presenting a small defect (panel $\mathrm{C}$ ), the pattern was similar. However, in the case of larger defect (panel D), CX staining was found in the interterritorial matrix and extended to the middle zone while oxPTM-CII staining extended to the middle and superficial zone. Interterritorial staining of oxPTM-CII was also observed in the middle and superficial zones in the area of the damaged fibrillar cartilage (panel D). In addition, in the middle and superficial zones, cells positive for oxPTM-CII but no CX were observed (see arrows). These results indicate that oxPTM-CII and CX staining mainly co-localize (around the larger chondrocytes in the deep zone) in early OA but might show a slightly different pattern at later OA stages. Finally, condylar cartilage facing a large defect (here picture from panels $\mathrm{D}$ and $\mathrm{E}$ are from the same animal) were positive for both staining and the staining were observed in all zones of cartilage.
The area of the cartilage positive for oxPTM-CII and CX increases with the progression of the disease

The areas positively stained for oxPTM-CII and CX were quantified in the medial tibial cartilage for both models (Fig. 4). In the DMM model, the oxPTM-CII and CX positive areas were larger in the operated knee compared to the contralateral knee and their size increased with disease progression. Compared to contralateral knees, this increase was not significant at early time points and became significant at day 28 for oxPTM-CII and days 14 and 28 for CX. These results strengthen the previous observations that oxPTM-CII and $\mathrm{CX}$ extend progressively beyond the deep zone as OA progresses.

For the ACLT+pMx model, the stained area for oxPTM-CII remained small, and similarly to the DMM model no difference to the contralateral medial cartilage could be observed at day 14 or before. However, a trend to increased oxPTM-CII can be observed at day $14(p=0.0920)$. The CX staining was found to be highly variable. There was a trend to an increased positive area at day 3 to 14 (only significant at day 5) but no clear progression can be observed. These results are in accordance with the
oxPTMCII

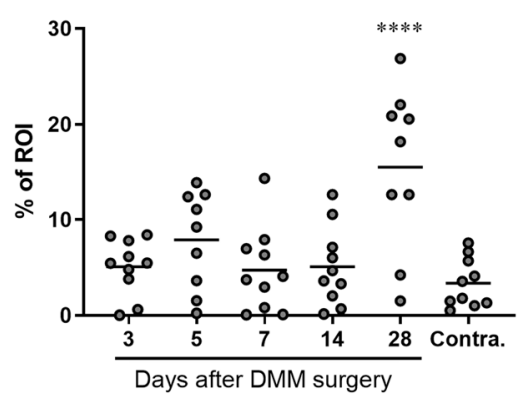

OxPTMCII

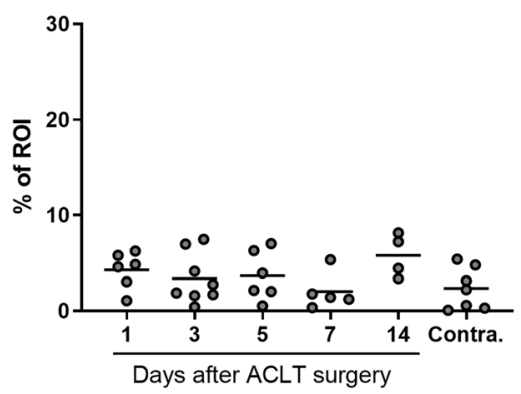

CX

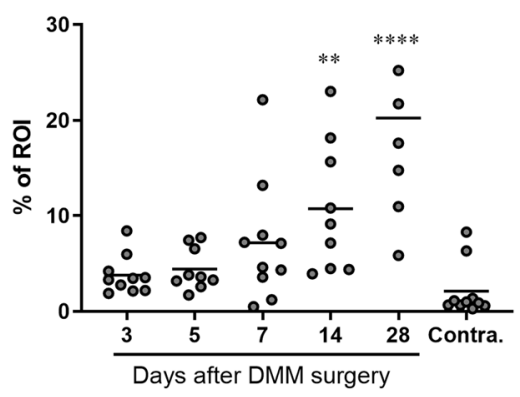

CX

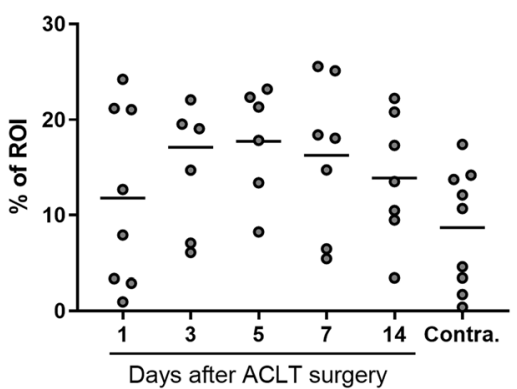

Fig. 4 Quantification of oxPTM-CII and CX stainings in the cartilage of the medial tibial plateau in the DMM and ACLT+tMx models. A region of interest (ROI) was defined in the medial tibial plateau and the area stained in blue (for CX) or in brown (for oxPTM-CII) was measured and normalized by the total cartilage area in the ROI. Data on the graphs represent the \% area of the ROI for each animal $(\mathrm{N}=4-10)$ and the mean for each time point and for the selected contralateral knees. A single asterisk, double asterisks, and quadruple asterisks mean significantly different from contralateral with $p<0.05,0.01$, or 0.0001 , respectively 
histological score (Fig. 1) which did not progress for the ACLT+pMx study between day 1 and 14 .

Finally, it would have been interesting to compare the operated knees to healthy rat knees instead of collateral knee of OA rats, to evaluate if a significant difference for oxPTM-CII and CX can appear at earlier time points. Indeed, in this study, the contralateral knees were selected from all time points ( 2 rats per time point) and as we described above, the positive areas for oxPTM-CII and CX started to increase contralateral knees at day 14 for the ACLT+pMx and day 28 for the DMM models. This would suggest that for this readout contralateral knees are not equivalent to those of healthy controls.

\section{The growth plate and the meniscus are positive for oxPTM-CII}

As expected, the growth plate and the calcified part of the meniscus were positive for CX (Fig. 5). These tissues were also found to be positive for oxPTM-CII. In these two tissues, both staining appear to co-localize.

\section{Discussion}

The ACLT+pMx (or tMx) and DMM models are both surgically induced instability OA models that are broadly used to study disease progression. In the present study, we investigated early disease progression for both models and evaluated the presence of oxidized type II collagen in cartilage and its co-localization with type $\mathrm{X}$ collagen-a marker of chondrocyte hypertrophy.

In the current study, we used an ACLT+pMx and DMM model in rats housed in colony cages enabling free movement of the animals $[9,16]$. In accordance with previous results from others [9], the total histopathological scores were slightly higher with the ACLT+ $\mathrm{pMx}$ model than the DMM model for the same time points and the diseased developed earlier with the
ACLT+pMx. Prolonging both studies for a longer time could have enabled to observe bigger difference between both models [20]. Interestingly, when looking at the subscores, the ACLT+pMx influenced only two categories (cartilage structure and matrix staining) until day 14, whereas after DMM all sub-scores were affected.

Oxidative stress is known to play a major role in OA [2, 21]. To investigate if oxidative stress is an early or late event in OA and to better understand how it affects cartilage and chondrocytes, we used an antibody against oxidized type II collagen (oxPTM-CII). Anti-oxPTM-CII was developed to recognize different forms of oxidized type II collagen and was demonstrated to bind human OA and RA cartilage, but not healthy cartilage [12]. OxPTM-CII was detected in the medial tibial cartilage of the operated knees as early as 1 and 3 days after ACTL+pMx and DMM surgeries, respectively, and before any cartilage damage was visible. Until 14 days post-surgery, the staining localized in the deep zone in the pericellular and territorial matrix of large hypertrophic chondrocytes. When larger defects occurred however, oxPTM-CII extended to the interterritorial matrix through the complete depth of cartilage. The staining was absent from the lateral tibial and femoral condylar cartilage but with the progression of the disease, the staining became visible in both these compartments (day 14 for ACLT+pMx, day 28 for DMM). This is in accordance with the observations from others $[9,22]$ that in these OA models, lesions develop primarily in the medial tibial plateau. We also looked at the contralateral knees and found that oxPTM-CII staining was absent or weak at early time points but started to appear at later time points. This finding suggests that the disease also starts to develop in the contralateral knee, possibly because the animal changes their gait thus inducing mechanical stress in the contralateral joint [23]. This is also expected to be accelerated in the colony housing where

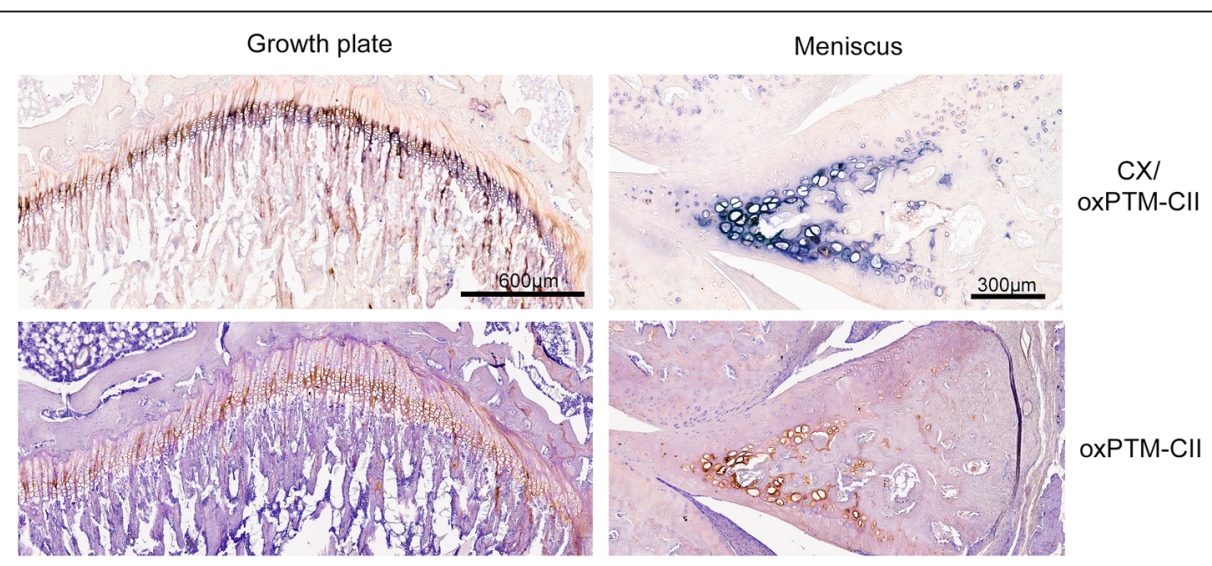

Fig. 5 CX and OXPTM-CIl staining in the meniscus and in the growth plate. One example of a double staining for type $X$ collagen (blue, CX) and oxPTM type II collagen (brown, oxPTM-CII) and the single staining for oxPTM-CII is shown for the growth plate and the meniscus 
animal are free to perform more weight-bearing activities compared to smaller cages $[16,20]$. It was already demonstrated that under mechanical stress chondrocytes produce ROS [24]. It is also known that removal or displacement of the meniscus increases peak stress in the medial compartment [25] and that cartilage normally covered by the meniscus possesses decreased load-bearing capacity and less resilience to damage compared to cartilage not covered by menisci [26]. We postulate that the DMM and the ACLT+pMx surgeries produce a strong mechanical stress in the tibial cartilage that was covered by the meniscus before the surgery resulting in ROS production [2] and the subsequent appearance of oxidized type II collagen. The localization of the staining likely corresponds to the zone where mechanical stress was the highest. It is also interesting to note that on the lateral compartment where the meniscus remained, and consequently mechanical stress was lower, the staining was more diffuse and not restricted to the deep zone. A similar pattern was observed in the medial condyles. Possibly, in medial tibial cartilage, the production of ROS and the resulting oxPTM-CII arises from excessive loading while on the lateral side and the condyles the ROS production and oxPTM-CII staining might be rather due to the diffusion of ROS and other inflammatory components from the medial tibial cartilage to other joint compartments.

Because oxPTM-CII was predominantly found in the deep zone and given that ROS are known to stimulate chondrocyte hypertrophy $[27,28]$, we also evaluated if oxPTM-CII co-localizes with type X collagen (CX). Indeed, we observed a strong co-localization and both staining became more intense with disease progression. We also observed that 28 days after DMM surgery, CX staining extended to the complete depth of cartilage at a time when large defects were also observed, resulting in a larger area positively stained for CX. Previous studies also described an increased CX expression during OA $[29,30]$ and in line with our observation, a pericellular staining in the deep zone and in advanced OA also in the middle zone was reported [30]. However, to our knowledge, the present study is the first that shows an increase of CX expression early in the disease. Similarly to oxPTM-CII, CX staining was detected before any apparent cartilage damage develops. At later time points (14 days for ACLT+pMx and 28 days for DMM), staining no longer fully co-localized. The co-localization was still found in the deep zone as observed at earlier time points but staining only partially co-localized in the middle and the superficial zone. This finding demonstrates that the formation of oxPTM-CII was not restricted to hypertrophic cells. We hypothesize that chondrocytes first produce ROS, which induces hypertrophy $[27,28]$ and subsequently lead to CX deposition. Possibly cells from the middle zone and superficial layer are more resistant to hypertrophy [31] increasing the delay between ROS production and CX production in these zones. However, the chondrocytes in this zone might have already been pre-hypertrophic and with this in mind it would have been of interest to investigate the presence of Runx2, an early hypertrophy marker. Taken together, our results confirm the therapeutic potential of inhibiting ROS to treat OA. Indeed, it has already been demonstrated that oral administration of the anti-oxidant $\mathrm{N}$-acetyl cysteine (NAC) protects against OA in the rat [32] and reduces hypertrophy in the growth plate of mice [28]. Future studies could use NAC to evaluate its impact on oxPTM-CII generation, hypertrophy, and OA initiation and progression in animal models.

Interestingly, the quantification of the medial tibial cartilage area positive for oxPTM-CII and CX revealed that in the DMM model, the CX staining spread across a larger cartilage area at day 14 already when compared with day 28 for oxPTM-CII. This may indicate that beside ROS production other mechanisms might induce cartilage hypertrophy in OA.

In addition, our results are in accordance with previous observations that the localization of oxPTM-CII is predominantly in the ipsilateral joint and that it is detected ahead of cartilage structural changes [12, 13]. In previous work, we used Cy5.5-anti-ROS CII antibody or Cy5.5-anti-ROS CII scFv injected i.v. or i.a. to detect OA changes in vivo with non-invasive imaging in DMM mice after 4 or 8 weeks. The present study demonstrates that labeled oxPTM-CII antibody or scFv could diagnose OA even earlier in the DMM model and could be used to monitor disease progression. Future longitudinal studies will need to assess the utility of anti-oxPTM-CII as a novel molecular imaging tool to both detect early onset and to longitudinally monitor $\mathrm{OA}$ in small animal models. In addition, because at early time points the oxPTM-CII staining was restricted to few cells in the deep zone, the anti-oxPTM-CII might need to be optimized to diffuse efficiently in cartilage and provide a high signal-to-noise ratio. If proven to be successful, anti-oxPTM-CII may be exploited for molecular imaging in parallel with future developments of MRI capabilities in human. Imaging with anti-oxPTM-CII may be interpreted in association with MRI/radiography for enhanced overall clinical management of patients, as well as improvement of outcome readouts in clinical trials.

In conclusion, oxPTM-CII and CX staining of ACLT+ pMx and DMM rat knees showed that the disease starts extremely early (day 1 and 3, respectively) in the deep zone of tibial medial cartilage and that the load-bearing zone that was covered by the meniscus before surgery was affected first. Our results confirm that chondrocyte hypertrophy is an integral part of the OA pathobiology, 
and we propose that it might be an initiating event of the disease. In addition, because oxPTM-CII and CX staining were strictly localized in the pericellular matrix at early time points, this study also supports the hypothesis that $\mathrm{OA}$ is a disease of the pericellular matrix [33,34].

\section{Conclusions}

We propose that oxPTM-CII antibodies or oxPTM-CII $\mathrm{scFv}$ labeled with a fluorescent probe is a promising biomarker to detect OA initiation ahead of radiographic changes and monitor its progression.

\section{Abbreviations}

OA: Osteoarthritis; Cll: Collagen type II; OxPTM-Cll: Oxidative posttranslationally modified collagen type II; CX: Collagen type X; ACLT+pMx: Anterior cruciate ligament transection with partial meniscectomy; DMM: Destabilization of the medial meniscus; ROS: Reactive oxidants

\section{Supplementary Information}

Supplementary information accompanies this paper at https://doi.org/10. 1186/s13075-021-02502-1.

Additional file 1: Table S1. Sub-scores for the histological scoring of OA. Figure S1. Histological sub-scores for the medial tibial plateau in the DMM model. Rats underwent DMM surgery and were sacrificed at days 3, $5,7,14$ and 28. The ipsilateral or contralateral knees were taken for histological analysis. Slides were stained with toluidine blue and saffron du Gatinais and evaluated according to the sub-scores detailed in the Table S1. Individual data for each animal $(N=9-10)$ and the mean is shown for each timepoint as well as for the selected contralateral knees. ${ }^{*}{ }^{* *}$ and

*** means significantly different from contralateral with $p<0.05, p<0.01$ or $p<0.001$ respectively. Figure S2. Histological sub-scores for the medial tibial plateau in the ACLT+pMx model. Rats underwent ACLT+pMx surgery and were sacrificed at days 1, 3, 5,7 and 14. The ipsilateral or contralateral knees were taken for histological analysis. Slides were stained with toluidine blue and saffron du Gatinais and evaluated according to the sub-scores detailed in the Table S1. Individual data for each animal $(N=9-10)$ and the mean is shown for each timepoint as well as for the selected contralateral knees. ${ }^{*},{ }^{* *},{ }^{* * *}$ and ${ }^{* * * *}$ means significantly different from contralateral with $p<0.05,0.01,0.001$, and 0.0001 respectively. The sub-score chondroosteophyte is not shown because it was equal to 0 in all groups. Figure S3. Staining and total histological score for the lateral tibial plateaus in the ACLT+pMx and DMM models. A. At various timepoints, the knees were taken for histological analysis and stained with toluidine blue and saffron du Gatinais or for type X collagen (blue, CX) and oxPTM type II collagen (brown, OXPTM-CII). Staining for the lateral tibial plateau for different timepoints and scores obtained for the medial plateau (in brackets) are shown. B. The total histological score for the lateral tibial plateau was determined according to the sub-scores detailed in the Table S1. Individual data for each animal $(N=9-10)$ and the mean is shown for each timepoint and selected contralateral knees. * means significantly different from contralateral with $p<0.05$. Figure S4. Staining of the contralateral tibial plateaus in the ACLT+pMx and DMM models. Representative pictures obtained with the toluidine blue and saffron du Gatinais staining, type X collagen (in blue, CX) and oxPTM-type ॥ collagen (in brown, oxPTM-CII) single or double immunostainings are shown.

\section{Acknowledgements}

The authors thank Andreas Westhof, Herbert Ziegler, Jennifer Freiwald, Julianne Dalchow, and Nicole Ellinghaus who contributed to this work.

\section{Authors' contributions}

All authors contributed to the study conception and design. Ahuva Nissim and Louise Topping provided the oxPTM-Cll antibody, Christian Brenneis organized the in vivo studies. Donata Harazin optimized and performed the staining and Didier Merciris performed the quantification of the oxPTM-Cll and CX immunohistochemistry stainings. Sven Lindemann supervised the histology work and helaped at critically intrepretig the data. Anne Gigout, Ahuva Nissim, and Louise Topping drafted the manuscript which was critically reviewed by all authors. All authors have read and approved the final submitted manuscript.

\section{Funding}

No funding was received for this article.

\section{Availability of data and materials}

All data generated during this study are included in this published article and in its supplementary information files or are available from the corresponding author on reasonable request.

\section{Declarations}

Ethics approval and consent to participate

All procedures were approved by the animal protection authorities of the local district government (Regional Authorities of Hessen, Germany, approval number DA 4/1019, approved on 29 March 2017).

\section{Consent for publication}

Not applicable.

\section{Competing interests}

Anne Gigout, Sven Lindemann, Christian Brenneis, and Donata Harazin were employees of Merck KGaA at the time of the study. Anne Gigout and Didier Merciris are currently employees of Galapagos SASU. The other authors declare that they have no competing interests.

\section{Author details}

'Osteoarthritis Research, Merck KGaA, Darmstadt, Germany. ${ }^{2}$ Barts and the London School of Medicine and Dentistry, Queen Mary University of London, Chaterhouse Square, London EC1M 6BQ, UK. ${ }^{3}$ Galapagos SASU, Romainville, France.

Received: 22 July 2020 Accepted: 3 April 2021

Published online: 14 April 2021

\section{References}

1. Loeser RF, Goldring SR, Scanzello CR, Goldring MB. Osteoarthritis: a disease of the joint as an organ. Arthritis Rheum. 2012;64(6):1697-707. https://doi. org/10.1002/art.34453.

2. Bolduc JA, Collins JA, Loeser RF. Reactive oxygen species, aging and articular cartilage homeostasis. Free Radic Biol Med. 2019;132:73-82. https:// doi.org/10.1016/j.freeradbiomed.2018.08.038.

3. Liu-Bryan R, Terkeltaub R. Emerging regulators of the inflammatory process in osteoarthritis. Nature Rev Rheum. 2015;11(1):35-44. https://doi.org/10.103 8/nrrheum.2014.162

4. Collins JA, Diekman BO, Loeser RF. Targeting aging for disease modification in osteoarthritis. Curr Opin Rheumatol. 2018;30(1):101-7. https://doi.org/10.1 097/BOR.0000000000000456.

5. Henrotin YE, Bruckner $P$, Pujol JP. The role of reactive oxygen species in homeostasis and degradation of cartilage. Osteoarthr Cartil. 2003;11(10): 747-55. https://doi.org/10.1016/S1063-4584(03)00150-X.

6. Carlo MD Jr, Loeser RF. Increased oxidative stress with aging reduces chondrocyte survival: correlation with intracellular glutathione levels. Arthritis Rheum. 2003;48(12):3419-30. https://doi.org/10.1002/art.11338.

7. Cope PJ, Ourradi K, Li Y, Sharif M. Models of osteoarthritis: the good, the bad and the promising. Osteoarthr Cartil. 2019;27(2):230-9. https://doi.org/1 0.1016/j.joca.2018.09.016.

8. Aizah N, Chong PP, Kamarul T. Early alterations of subchondral bone in the rat anterior cruciate ligament transection model of osteoarthritis. Cartilage. 2019:1947603519878479. https://doi.org/10.1177/1947603519878479.

9. Glasson SS, Blanchet TJ, Morris EA. The surgical destabilization of the medial meniscus (DMM) model of osteoarthritis in the 129/SvEv mouse. Osteoarthr Cartil. 2007;15(9):1061-9. https://doi.org/10.1016/j.joca.2007.03.006.

10. Inglis J, McNamee KE, Chia SL, Essex D, Feldmann M, Williams RO, et al. Regulation of pain sensitivity in experimental osteoarthritis by the 
endogenous peripheral opioid system. Arthritis Rheum. 2008;58(10):3110-9. https://doi.org/10.1002/art.23870.

11. Little CB, Barai A, Burkhardt D, Smith SM, Fosang AJ, Werb Z, Shah M, Thompson EW. Matrix metalloproteinase 13-deficient mice are resistant to osteoarthritic cartilage erosion but not chondrocyte hypertrophy or osteophyte development. Arthritis Rheum. 2009;60(12):3723-33. https://doi. org/10.1002/art.25002.

12. Hughes C, Faurholm B, Dell'Accio F, Manzo A, Seed M, Eltawil N, Marrelli A, Gould D, Subang C, al-Kashi A, de Bari C, Winyard P, Chernajovsky Y, Nissim A. Human single-chain variable fragment that specifically targets arthritic cartilage. Arthritis Rheum. 2010;62(4):1007-16. https://doi.org/10.1002/art.27346.

13. Lim NH, Vincent TL, Nissim A. In vivo optical imaging of early osteoarthritis using an antibody specific to damaged arthritic cartilage. Arthritis Res Ther. 2015;17(1):376. https://doi.org/10.1186/s13075-015-0898-5.

14. Topping LM, Thomas BL, Rhys HI, Tremoleda JL, Foster M, Seed M, Voisin MB, Vinci C, Law HL, Perretti M, Norling LV, Azevedo HS, Nissim A. Targeting extracellular vesicles to the arthritic joint using a damaged cartilage-specific antibody. Front Immunol. 2020;11:10. https://doi.org/1 0.3389/fimmu.2020.00010

15. Harrison JL, Williams SC, Winter G, Nissim A. Screening of phage antibody libraries. Methods Enzymol. 1996;267:83-109. https://doi.org/10.1016/S00766879(96)67007-4.

16. Brenneis C, Westhof A, Holschbach J, Michaelis M, Guehring H, Kleinschmidt-Doerr K. Automated tracking of motion and body weight for objective monitoring of rats in colony housing. J Am Assoc Lab Anim Sci. 2017;56(1):18-31.

17. Kraus VB, Huebner UL, DeGroot J, Bendele A. The OARSI histopathology initiative - recommendations for histological assessments of osteoarthritis in the guinea pig. Osteoarthr Cartil. 2010;18(Suppl 3):S35-52. https://doi.org/1 0.1016/j.joca.2010.04.015.

18. Ramos-Vara JA, Beissenherz ME. Optimization of immunohistochemical methods using two different antigen retrieval methods on formalin-fixed paraffin-embedded tissues: experience with 63 markers. J Vet Diagn Investig. 2000;12(4):307-11. https://doi.org/10.1177/104063870001200402.

19. Dell'accio F, De Bari C, Eltawil NM, Vanhummelen P, Pitzalis C. Identification of the molecular response of articular cartilage to injury, by microarray screening: Wnt-16 expression and signaling after injury and in osteoarthritis. Arthritis Rheum. 2008;58(5):1410-21. https://doi.org/10.1002/art.23444.

20. Brenneis C, Menges S, Westhof A, Lindemann S, Thudium CS, KleinschmidtDoerr K. Colony housing promotes structural and functional changes during surgically induced osteoarthritis in rats. Osteoarthritis Cartilage Open. 2020; 2. https://doi.org/10.1016/j.ocarto.2020.100100.

21. Abramson SB. Osteoarthritis and nitric oxide. Osteoarthr Cartil. 2008; 16(Suppl 2):S15-20. https://doi.org/10.1016/S1063-4584(08)60008-4.

22. lijima H, Aoyama T, Ito A, Tajino J, Nagai M, Zhang X, Yamaguchi S, Akiyama H, Kuroki H. Destabilization of the medial meniscus leads to subchondral bone defects and site-specific cartilage degeneration in an experimental rat model. Osteoarthr Cartil. 2014;22(7):1036-43. https://doi.org/10.1016/j.joca.2 014.05.009.

23. Zhu J, Zhu Y, Xiao W, Hu Y, Li Y. Instability and excessive mechanical loading mediate subchondral bone changes to induce osteoarthritis. Ann Transl Med. 2020;8(6):350. https://doi.org/10.21037/atm.2020.02.103.

24. Goodwin W, McCabe D, Sauter E, Reese E, Walter M, Buckwalter JA, Martin JA. Rotenone prevents impact-induced chondrocyte death. J Orthop Res. 2010;28(8):1057-63. https://doi.org/10.1002/jor.21091.

25. Arunakul M, Tochigi Y, Goetz JE, Diestelmeier BW, Heiner AD, Rudert J, Fredericks DC, Brown TD, McKinley TO. Replication of chronic abnormal cartilage loading by medial meniscus destabilization for modeling osteoarthritis in the rabbit knee in vivo. J Orthop Res. 2013;31(10):1555-60. https://doi.org/10.1002/jor.22393.

26. Yeow CH, Lau ST, Lee PV, Goh JC. Damage and degenerative changes in menisci-covered and exposed tibial osteochondral regions after simulated landing impact compression-a porcine study. J Orthop Res. 2009;27(8):11008. https://doi.org/10.1002/jor.20861.

27. Kim KS, Choi HW, Yoon HE, Kim IY. Reactive oxygen species generated by NADPH oxidase 2 and 4 are required for chondrogenic differentiation. J Biol Chem. 2010;285(51):40294-302. https://doi.org/10.1074/jbc.M110.126821.

28. Morita K, Miyamoto T, Fujita N, Kubota Y, Ito K, Takubo K, Miyamoto K, Ninomiya K, Suzuki T, Iwasaki R, Yagi M, Takaishi H, Toyama Y, Suda T. Reactive oxygen species induce chondrocyte hypertrophy in endochondral ossification. J Exp Med. 2007;204(7):1613-23. https://doi. org/10.1084/jem.20062525.

29. Aigner T, Reichenberger E, Bertling W, Kirsch T, Stoss H, von der Mark K. Type $X$ collagen expression in osteoarthritic and rheumatoid articular cartilage. Virchows Arch B Cell Pathol Incl Mol Pathol. 1993;63(4):205-11. https://doi.org/10.1007/BF02899263.

30. Boos N, Nerlich AG, Wiest I, von der Mark K, Ganz R, Aebi M. Immunohistochemical analysis of type-X-collagen expression in osteoarthritis of the hip joint. J Orthop Res. 1999;17(4):495-502. https://doi. org/10.1002/jor.1100170406.

31. Jiang J, Leong NL, Mung JC, Hidaka C, Lu HH. Interaction between zonal populations of articular chondrocytes suppresses chondrocyte mineralization and this process is mediated by PTHrP. Osteoarthr Cartil. 2008;16(1):70-82. https://doi.org/10.1016/j.joca.2007.05.014.

32. Kaneko $Y$, Tanigawa $N$, Sato $Y$, Kobayashi T, Nakamura S, Ito E, Soma T, Miyamoto K, Kobayashi S, Harato K, Matsumoto M, Nakamura M, Niki Y, Miyamoto T. Oral administration of $\mathrm{N}$-acetyl cysteine prevents osteoarthritis development and progression in a rat model. Sci Rep. 2019;9(1):18741. https://doi.org/10.1038/s41598-019-55297-2.

33. Chery DR, Han B, Li Q, Zhou Y, Heo SJ, Kwok B, et al. Early changes in cartilage pericellular matrix micromechanobiology portend the onset of post-traumatic osteoarthritis. Acta Biomater. 2020;

34. Guilak F, Nims RJ, Dicks A, Wu CL, Meulenbelt I. Osteoarthritis as a disease of the cartilage pericellular matrix. Matrix Biol. 2018;71-72:40-50.

\section{Publisher's Note}

Springer Nature remains neutral with regard to jurisdictional claims in published maps and institutional affiliations.

\section{Ready to submit your research? Choose BMC and benefit from:}

- fast, convenient online submission

- thorough peer review by experienced researchers in your field

- rapid publication on acceptance

- support for research data, including large and complex data types

- gold Open Access which fosters wider collaboration and increased citations

- maximum visibility for your research: over $100 \mathrm{M}$ website views per year

At BMC, research is always in progress.

Learn more biomedcentral.com/submissions 